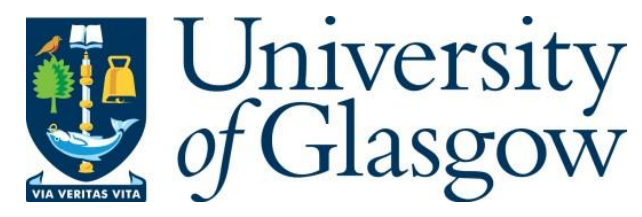

Alshuhri, M. S. , Gallagher, L., McCabe, C. and Holmes, W. M. (2020) Change in CSF dynamics responsible for ICP elevation after ischemic stroke in rats: a new mechanism for unexplained END? Translational Stroke Research, 11, pp. 310-318.

(doi: 10.1007/s12975-019-00719-6)

There may be differences between this version and the published version. You are advised to consult the publisher's version if you wish to cite from it.

http://eprints.gla.ac.uk/190426/

Deposited on 17 July 2019

Enlighten - Research publications by members of the University of Glasgow http://eprints.gla.ac.uk 


\section{Change in CSF dynamics responsible for ICP elevation after ischemic stroke in rats: A new mechanism for unexplained END?}

Mohammed S. Alshuhri ${ }^{1,2}$, MSc; Lindsay Gallagher ${ }^{1}$, HNC; Christopher McCabe ${ }^{1}$, PhD; William M. Holmes ${ }^{1}$, PhD

1. Glasgow Experimental MRI Centre (GEMRIC) (M.S.A., L.G., C.M., W.M.H), Institute of Neuroscience and Psychology, Garscube Estate, University of Glasgow, Glasgow, Scotland, United Kingdom.

2. Radiology and Medical Imaging Department, College of Applied Medical Sciences, Prince Sattam bin Abdulaziz University, Kharj, Saudi Arabia,

Corresponding Author: Dr William Holmes, Glasgow Experimental MRI Centre, Wellcome Surgical Institute, Garscube Estate, University of Glasgow, Glasgow, Scotland, United Kingdom, G61 1QH. Tel: ++44 1413306984. Email: William.Holmes@glasgow.ac.uk

\footnotetext{
Abstract

It has been proposed that intracranial pressure (ICP) elevation and collateral failure are responsible for unexplained early neurological deterioration (END) in stroke. The study's aims were to investigate whether cerebral spinal fluid (CSF) dynamics, rather than edema, are responsible for elevation of ICP after ischemic stroke. Permanent middle cerebral artery occlusion (pMCAO) was induced with an intraluminal filament. At $24 \mathrm{hrs}$ after stroke, baseline ICP was measured and CSF dynamics were probed via a steady-state infusion method. Diffusion weighted imaging (DWI) and T2 weighted magnetic resonance imaging were performed to define cerebral ischemic damage and the volume of brain swelling. We found that the pMCAO group exhibited a significant increase in CSF outflow resistance $(2.27 \pm 0.15 \mathrm{mmHg} \cdot \mu \mathrm{L}-1 . \mathrm{min})$ compared with the sham group $(0.93 \pm 0.06 \mathrm{mmHg} \cdot \mu \mathrm{L}-1 . \mathrm{min}, \mathrm{p}=0.002)$. There was no correlation between mean ICP at $24 \mathrm{hrs}$ post-pMCAO and edema $\left(\mathrm{r}^{2}=-0.03, \mathrm{p}=0.5\right)$ or infarct volumes $\left(\mathrm{r}^{2}=0.09, \mathrm{p}=0.5\right)$. However, for the first time, we found a significant correlation between the baseline ICP at $24 \mathrm{hrs}$ post-stroke and the value of CSF outflow resistance. Results show that CSF outflow resistance, rather than edema, was the mechanism responsible for ICP elevation following ischemic stroke. This challenges current concepts and suggests the possibility that intracranial hypertension may be occurring undetected in a much wider
} 
range of stroke patients than is currently considered to be the case. In addition, this further supports the hypothesis that unexplained Early Neurological Deterioration is the result of elevated ICP, leading to reduced collateral flow and cerebral perfusion.

Key words: intracranial pressure, early neurological deterioration, ischemic stroke, cerebrospinal fluid, MRI 


\section{Introduction}

Early neurological deterioration (END) can occur within the first 24-48 hours following acute ischemic stroke, with prevalence being relatively common (>14\%), and is strongly associated with poor outcome [1]. END was defined as an increase in the National Institutes of Health Stroke Scale (NIHSS) score of > 4 points within the first $24 \mathrm{hrs}$ after admission [2]. Although some cases of END have a straightforward cause, such as symptomatic intracranial hemorrhage and malignant edema, approximately $\sim 2 / 3$ of cases remain unexplained [3]. These unexplained END cases are associated with persistent proximal occlusion, large diffusion-perfusion mismatch, and diffusion weighted imaging lesion growth beyond the initial ischemic penumbra [3]. Several mechanisms have been proposed, involving either compromised hemodynamics (thrombus extension [1], collateral failure [4], reduced mean arterial pressure [5]) or other tissue-based mechanisms (excitotoxicity [6], inflammation [7], hyperthermia [8], edema [9], hyperglycemia [3], hypoxia [10]). Although these mechanisms may make some contribution, there is a lack of strong evidence associating them with END. An intriguing alternative mechanism has recently been proposed suggesting that elevated intracranial pressure (ICP), resulting in reduced collateral flow and cerebral perfusion, may be responsible for unexplained END [11]. However, the mechanism for this rise in ICP is currently unknown.

Conventionally, only patients with large malignant strokes are considered to be at risk of elevated ICP, due to space occupying vasogenic edema within the closed cranial compartment. This logic has been extended to infer that ICP does not become elevated in cases of smaller, non-malignant strokes, since there is little or no vasogenic edema. However, we can find no evidence in the clinical literature to support this broad assumption, presumably because the risks associated with invasive ICP measurement prevent its use.

This assumption is also contradicted by recent animal experiments, which show dramatic increases in ICP occurring around $24 \mathrm{hrs}$ after stroke onset, even following small strokes where there is little or no vasogenic edema [12]. This evidence leads us to hypothesize that a mechanism, other than edema, is responsible for ICP elevation in these cases. Inspection of a mathematical model of ICP indicates that changes in the cerebral spinal fluid (CSF) dynamics may be responsible for the observed increases in ICP post-ischemic stroke [13,14]. Therefore, in the present study, we have investigated the relationship between CSF dynamics, vasogenic edema, and ICP following experimental stroke. 


\section{Materials and Methods}

\section{Animals and experimental protocol}

Experiments were carried out under license from the UK Home Office in accordance with the Animals (Scientific Procedures) Act, 1986, incorporating European Directive 2010/63/EU and approved by the University of Glasgow Ethical Review Panel. Male Wistar Kyoto (WKY) rats (350 to 400 g, 24 to 32 weeks old) were initially anesthetized (5\% isoflurane in 20:80 $\mathrm{O}_{2}$ /air mixture) in an induction chamber, then intubated, and artificially ventilated (with 2$3 \%$ isoflurane in 20:80 $\mathrm{O}_{2}$ /air mixture at flow rate 1-2 L/min). Body temperature was monitored throughout surgery with a rectal thermocouple and maintained at $37 \pm 0.5^{\circ} \mathrm{C}$. The femoral artery was cannulated with PE-50 tubing for continuous monitoring of arterial blood pressure and heart rate (Biopac Systems, MP100) and for the measurement of arterial blood gases (Bayer, Rapidlab 248). Animals were randomly allocated to either a permanent middle cerebral occlusion group ( $\mathrm{pMCAO}, \mathrm{n}=8$ ) or a sham surgery group (sham, $\mathrm{n}=6$ ).

\section{Middle Cerebral Artery Occlusion}

Permanent occlusion of the left MCA was performed by intraluminal thread occlusion, as previously described [15].

The left common carotid artery was isolated and an arteriotomy was performed to allow for the insertion of a 4-0 nylon silicone coated tip monofilament (403934PK10 or 404134PK10 Doccol Corporation, USA) through the internal carotid artery until it blocked the origin of the MCA. The filament was left in place to induce a pMCAO. For sham group rats, surgery to reveal the MCA was carried out but the artery was not occluded. Immediately following pMCAO, rats were transferred to the MRI scanner and diffusion weighted imaging (DWI) was conducted to assess baseline lesion volume. Rats were then recovered from the anaesthesia and, $24 \mathrm{hrs}$ after pMCAO, reanaesthetised for assessment of final infarct volume using T2 weighted MRI. The animal was then transferred into the surgery room for ICP measurements. The experimental protocol is shown schematically in Fig. 1. 


\section{Intracranial pressure (ICP) measurement}

Following MRI scanning at 24hrs post-MCAO or sham surgery, rats were re-anesthetized (5\% isoflurane in 20:80 $\mathrm{O}_{2} /$ air mixture) and transferred to a stereotaxic frame where the head was secured in ear and tooth bars. The coordinates (Bregma $1.3 \mathrm{~mm}$ posterior, $2 \mathrm{~mm}$ lateral) for the lateral ventricles were identified from the rat brain stereotaxic atlas (Paxinos \& Watson) and, where necessary, adjusted slightly to accommodate any brain swelling identified on the $T_{2}$ weighted MRI scans. Two burr holes were drilled over the ipsilateral and contralateral lateral ventricles and, for ICP monitoring, a 25-gauge needle connected by an air-free fluid system was placed in the ipsilateral ventricle and attached to a pressure transducer for continuous ICP recording. The probe was secured to the skull with cyanoacrylate glue to avoid leakage. The correct position of the ICP probe within the ventricle was confirmed by the presence of CSF pulsation within the catheter. Prior to ICP recording, pressure transducers were calibrated with a Sphygmomanometer and the ICP waveform signal was confirmed by response to abdominal compression, ensuring clear cardiac and respiratory waveforms were visible.

\section{CSF infusion}

CSF dynamics were probed via a steady-state infusion method [16], involving stepwise increases in the intracerebroventricular infusion of artificial cerebrospinal fluid (aCSF) (Supplemental Figure. 1). A catheter was inserted into the contralateral lateral ventricle and secured to the skull with cyanoacrylate glue, then connected to an infusion pump (Graseby /3150 Syringe Pump). The baseline ICP pressure was monitored for 30 minutes before starting infusion of aCSF (aCSF: NaCl 140 mmol/L, KCl 3 mmol/L, NaH2PO4 12 mmol/L, NaHCO3 18 mmol/L, $\mathrm{CaCl} 22.5 \mathrm{mmol} / \mathrm{L}, \mathrm{pH}$ 7.4). The infusion rate was adjusted incrementally 1.67, 3.33, 5.00, 8.30, 11.67, 16.67, 25.00 and $33.3 \mu \mathrm{L} / \mathrm{min}$ ), with 3-6 minutes allowed for a steady-state ICP to be established after each increase. During the infusion experiment, mean arterial pressure (MAP) and ICP were continuously monitored in all rats. Blood gases $\left(\mathrm{pH}\right.$, partial pressure of oxygen $\left[\mathrm{P}_{\mathrm{a}} \mathrm{O}_{2}\right]$, carbon dioxide $\left[\mathrm{P}_{\mathrm{a}} \mathrm{CO}_{2}\right]$ were measured before and after the infusion. 


\section{Mathematical modeling}

Marmarou and colleagues [14] developed a mathematical model of the CSF/ICP system. Here we have extended their steady-state case to include growth in brain edema. In the closed cranial cavity, steady-state ICP exists when rates balance.

$$
Q_{\text {prod }}+Q_{\text {edema }}=Q_{a b s}
$$

Where, $Q_{\text {prod }}$ is the rate of CSF production and $Q$ edema is the rate of edema growth. Assuming the rate of CSF absorption across the arachnoid villi, $Q_{\text {abs, }}$ is proportional to the corresponding pressure drop $\Delta P=P_{I C P}-P_{S S}$, where the venous sagittal sinus pressure $\mathrm{P}_{\mathrm{ss}}$ is small, with a CSF outflow resistance R, gives

$$
P_{I C P}=R Q_{a b s}=R\left(Q_{\text {prod }}+Q_{\text {edema }}\right) \quad(\text { Equation } 2)
$$

In the case of the steady state infusion experiments, as the rate of infusion of aCSF is greater than $\left(Q_{p r o d}+\right.$ $\left.Q_{\text {edema }}\right)$, this becomes

$$
P_{I C P}=R Q_{a b s} \cong R Q_{\text {inf }}+c \quad \text { (Equation 3) }
$$

where $Q_{\text {inf }}$ is the rate of infusion of aCSF. It can be seen from equation 3 that the CSF outflow resistance is then obtained from the slope of the plot $\Delta P$ vs $Q_{\text {inf }}$.

\section{Magnetic Resonance Imaging and Analysis}

MRI data were acquired using a Bruker Pharmascan 7T/16cm system (Ettlingen, Germany) with a gradient coil insert (internal diameter $=90 \mathrm{~mm}, 300 \mathrm{mT} / \mathrm{m}$ ) and a four-channel phased-array surface receive coil used for brain imaging. During MRI scanning, a pilot sequence was first acquired to determine the correct geometry. A diffusion weighted imaging (DWI) scan (4-shot, spin-echo, EPI) was performed 30 minutes after MCAO, allowing quantitative apparent diffusion coefficient (ADC) maps to be produced (echo time $[\mathrm{TE}]=22 \mathrm{~ms}$, repetition time $[\mathrm{TR}]=4,000 \mathrm{~ms}$, matrix $=96 \times 96$, field of view $=25 \mathrm{~mm} \times 25 \mathrm{~mm}$, three directions $=\mathrm{x}, \mathrm{y}, \mathrm{z}, \mathrm{B}$ values $=0$ and $1,000 \mathrm{~s} / \mathrm{mm}^{2}$, eight coronal slices; $1.5 \mathrm{~mm}$ thickness). A T2-weighted (RARE) scan was performed to assess infarct 
volume at 24hrs (effective echo Time $[\mathrm{TE}]=46.6 \mathrm{~ms}$, repetition time $[\mathrm{TR}]=5,000 \mathrm{~ms}$, RARE factor 8, averages 2 , matrix $256 \times 256$, FOV $3.0 \mathrm{~cm} \times 3.0 \mathrm{~cm}, 8$ slices, $0.75 \mathrm{~mm}$ thickness).

\section{Data Analysis}

Quantitative ADC maps, in units of square millimeters per second, were calculated from the Stejskal-Tanner equation using Image J software (http://rsb.info. nih.gov/ij/. A 23\% reduction in mean contralateral ADC was used to determine ischemia lesion volume from the multi-slice ADC maps [18] (Supplemental Figure. 2). Infarct volume was determined at $24 \mathrm{hrs}$ from $\mathrm{T} 2$-weighted images by manual tracing of the hyperintense lesion area on each slice. This was then summed and multiplied by the slice thickness (Supplemental Figure. 3). The edema-corrected lesion volume (LVe) and the space occupying effect in percent of edema uncorrected lesion volume (SE) were calculated from equations derived by Gerriets [18]:

$$
\begin{gathered}
L V e=H V c+H V i-(H V c+H V i-L V u) \times \frac{H V c+H V i}{2 H V c} \quad \text { (Equation 4) } \\
S E=L V u-L V e
\end{gathered}
$$

$\mathrm{HVi}$ and $\mathrm{HVc}$ refer to the volumes of the ipsilateral and contralateral hemispheres, respectively. LVu represents the uncorrected lesion volume as delineated on the T2W images. All volumetric measurements were performed independently by two independent investigators. To examine interobserver reproducibility, investigator 2 determined infarct and edema volumes on the same images and the calculated values were compared with the results performed by investigator 1 . Any cases with $>10 \%$ discrepancy was flagged for review.

\section{Exclusion criteria}

A total of 14 Wistar Kyoto (WKY) rats were used in this series of experiments. Two animals in the MCAO group were excluded due to technical problems (CSF leaks, unstable BP and death). Hence, 12 animals were available for data analysis (6 in each group). 


\section{Impact of aCSF infusion on edema size}

To ensure that infusion of aCSF does not contribute to the development of brain edema, a pilot experiment was conducted with the same infusion rates and durations used in the original experiments (experimental details are described in Supplemental Figures 4 and 5). Serial T2 MRI revealed that there is no change in edema with aCSF infusion.

\section{Statistical analysis}

A sample size calculation was performed using pilot data ( $G$ *Power v.3.1.3 software) [19], which estimated that six animals per group were required to detect a $60 \%$ difference in CSF outflow resistance and $12 \mathrm{mmHg}$ difference in $\triangle \mathrm{ICP}$ between the sham and pMCAO groups with standard settings of alpha 0.05 , power 0.8 . Students t-tests were performed on physiological parameters for illustrative purposes to highlight changes between pMCAO and sham rats. Mann-Whitney U-tests were performed to compare mean ICP and CSF resistance values between sham and post-stroke groups. Spearman's correlation analysis was used to determine the correlation between ICP and edema volume, infarct volume and CSF resistance. For the CSF infusion test, statistical analysis was performed with the Wilcoxon signed-rank test on ICP, MAP and cerebral perfusion pressure (CPP) to detect differences between each infusion step point and baseline values. Data are presented as mean \pm standard deviation unless otherwise stated. All tests were considered statistically significant for $\mathrm{p}$ values $<0.05$.

\section{Results}

\section{ICP and Physiological variables}

ICP oscillation with pulse pressure and respiration was detected in all experiments (Fig. 2a). Median ICP at baseline in the pMCAO group at $24 \mathrm{hrs}(18.6 \pm 5.84 \mathrm{mmHg})$ was demonstrated to be significantly greater than the sham group $(6.01 \pm 0.56 \mathrm{mmHg})(\mathrm{p}=0.002)$ (Fig. 2b). Physiological variables before and after infusion of aCSF are shown in Table 1. MAP was slightly lower at baseline in the pMCAO rats compared with the sham operated rats, however, this difference was not statistically significant. CPP (MAP - ICP) was significantly lower at baseline in 
pMCAO rats compared with the sham operated rats $(65 \pm 10 \mathrm{mmHg}$ vs. $89 \pm 8 \mathrm{mmHg}, \mathrm{p}=0.049)$. There were no statistically significant differences between groups for intra-operative physiological indices with the exception of $\mathrm{P}_{\mathrm{a}} \mathrm{CO}_{2}$ (Table 1).

\section{Edema and Infarct volume vs. ICP}

Mean edema volume was $128.4 \pm 19.3 \mathrm{~mm}^{3}$ (range $100-146 \mathrm{~mm}^{3}$ ) at $24 \mathrm{hrs}$ post-MCAO (online-only Data Supplemental Figure. 2). The growth rate of edema formation $(0.09 \pm 0.01 \mu / \mathrm{min})$ was estimated by dividing the edema volume by 1,440 minutes (i.e. 24hrs). Mean edema-corrected infarct volume was $342.5 \pm 65 \mathrm{~mm}^{3}$ (range 225-397 mm3). There was no significant correlation between mean ICP at $24 \mathrm{hrs}$ and infarct volume $\left(\mathrm{r}^{2}=0.09, \mathrm{p}=\right.$ $0.5)$ or edema volume $\left(r^{2}=-0.03, p=0.5\right)$ (Figs. 3a and $\left.3 b\right)$.

\section{ICP response to aCSF infusion}

The time course for MAP, CPP and ICP changes after aCSF infusion in both pMCAO and sham groups is shown in Figure 4. After the start of aCSF infusion, ICP increased significantly ( $p<0.001$ for all time points) in both the pMCAO and sham group. Figure 5a shows how the steady state ICP changed from baseline ( $\triangle \mathrm{ICP})$ increases with increasing infusion rate. For both groups, plotting $\triangle \mathrm{ICP}$ versus infusion rate resulted in a linear relationship, in confirmation of equation 3. At the end of infusion, ICP dropped to the initial baseline value.

\section{CSF outflow resistance}

CSF outflow resistance was calculated for each animal, as in equation 3, from the initial slope of the $\triangle \mathrm{ICP}$ vs

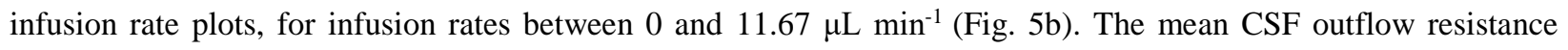
measured for the pMCAO group $\left(2.27 \pm 0.15 \mathrm{mmHg} \cdot \mu \mathrm{L}^{-1}\right.$.min $)$ was significantly greater than in the sham operated rats $\left(0.93 \pm 0.06 \mathrm{mmHg} \cdot \mu \mathrm{L}^{-1} \cdot \mathrm{min}\right)(\mathrm{p}=0.002)$ (Fig. 5c). A significant relationship was found between CSF outflow resistance and baseline ICP measured at $24 \mathrm{hrs}$, using both pMCAO and sham rats $\left(\mathrm{r}^{2}=0.85, \mathrm{p}<0.0001\right)$ (Fig. $\left.5 \mathrm{~d}\right)$. 


\section{CSF production rate}

Linear regression of the initial ICP verse infusion rate data to zero ICP (Fig. 5a) allowed an indirect estimation of CSF production rate, indicating no significant change in CSF production rate following stroke $(1.4 \pm 1.54 \mu \mathrm{L} / \mathrm{min}$ $\mu \mathrm{L} / \mathrm{min})$ compared to sham controls $(1.9 \pm 1.49 \mu \mathrm{L} / \mathrm{min})(\mathrm{p}=0.6)$.

\section{Discussion}

It is conventionally assumed that ICP elevation post-stroke is the result of cerebral vasogenic edema within the closed cranial compartment. Consequently, only patients with large malignant strokes and significant levels of brain edema are assumed to be affected by intracranial hypertension. Though this view has persisted over several decades, we can find no evidence in the clinical literature to support this broad assumption, presumably because the risks associated with invasive ICP measurements have precluded their use in the vast majority of stroke patients. Recently this assumption has been challenged by animal experiments, which show ICP can become substantially elevated at 24hrs, even following small ischemic strokes (sub-cortical lesions) where there is little or no 'space occupying' edema. Further, these previous studies found no correlation between edema volume and baseline ICP at $24 \mathrm{hrs}$ poststroke $[12,20]$. Data from the present study support these findings and also show an absence of correlation between edema volume and baseline ICP in the pMCAO group (Fig. 3) (Spearman correlation $r=-0.03, p=0.5$ ). However, since our power calculations were based on CSF outflow resistance pilot data, the sample size used $(n=6)$ is likely underpowered with regard to definitively determining a lack of correlation.

It has previously been proposed that increases in ICP in the first $24 \mathrm{hrs}$ post-ischemic stroke may provide an explanation for patients suffering a progressive stroke or unexplained END [21]. The effect of increasing ICP on collateral flow has been recently demonstrated in rats, using fluorescent microspheres to quantify flow through individual pial collateral vessels [22]. This showed progressive reductions in collateral flow with stepwise increases in ICP (up to $50 \%$ reduction in flow at $30 \mathrm{mmHg}$ ). Consistent with these earlier studies, we found even minor increases in ICP resulted in a decrease in the critical cerebral perfusion pressure (CPP) which is the driving pressure 
of the cerebral blood flow in the absence of autoregulation [23]. Although CPP is regulated by both MAP and ICP, it is remarkable how little attention the ICP component has received [23,24].

It has been suggested that any reductions in the already tenuous penumbral perfusion would be expected to impact penumbral survival, leading to late infarct expansion and END. Indeed, collateral failure has been associated with infarct growth and has been proposed as a potential mechanism of unexplained END [25]. In support of this, recent clinical imaging studies have shown infarct growth beyond the initial penumbra and the occurrence of "new DWI lesions" at day 7 post-stroke, outside the initial area of hypo-perfusion [26]. In addition, there have been numerous rodent studies of both permanent and transient MCAO which have shown infarct volume expanding beyond 24hrs $[27,28]$.

This raises the important question: if not edema, what mechanism is responsible for the substantial ICP elevations seen at $24 \mathrm{hrs}$ post-ischemic stroke? Inspection of the steady-state model of ICP (equation 2) shows ICP is dependent upon CSF outflow resistance, the rate of CSF production and the rate of edema growth. In confirmation of the mathematical model (equation 3), we found a linear relationship between the increase in ICP above baseline ( $\triangle \mathrm{ICP})$ and the infusion rate of aCSF (Fig. 5a) for both pMCAO and sham groups. Measured values of CSF outflow resistance for the sham group $\left(0.93 \pm 0.06 \mathrm{mmHg} . \mu \mathrm{L}^{-1}\right.$. $\left.\mathrm{min}\right)$ were consistent with previous measurements for rats, which ranged from 0.5 to $1.8 \mathrm{mmHg} . \mu \mathrm{L}^{-1} \cdot \min [29,30]$.

For the first time, we have shown that CSF outflow resistance increases significantly at $24 \mathrm{hrs}$ post-pMCAO compared with a sham operated group (Fig 5c). This more than a two-fold increase in CSF outflow resistance following ischemic stroke and is of a similar magnitude to increases previously encountered in studies on hydrocephalic rats [31]. Further, for the first time, we found a significant correlation between the baseline ICP at 24hrs post-pMCAO and the value of CSF outflow resistance (Fig. 5d).

The slow rate of CSF production is difficult to measure, with an estimate of the rate being made by extrapolating the initial ICP vs infusion data back to zero ICP. For the sham operated group this gave the rate of CSF production as $1.9 \pm 1.49 \mu \mathrm{L} / \mathrm{min}$, slightly lower than comparable data found in the literature $(3.7 \pm 0.1 \mu \mathrm{L} / \mathrm{min})$ measured using a ventriculo-cisternal perfusion technique [32]. We found the rate of CSF production did not significantly change in the pMCAO group $(1.4 \pm 1.54 \mu \mathrm{L} / \mathrm{min})$ compared to the sham group $(1.9 \pm 1.49 \mu \mathrm{L} / \mathrm{min})(\mathrm{p}=0.6)$. Moreover, in the pMCAO group, the rate of edema growth $(0.09 \pm 0.01 \mu \mathrm{L} / \mathrm{min})$ was found to be small compared with rate of CSF 
production. Taken together, these results indicate the ICP elevation seen at 24hrs post-pMCAO was primarily driven by increased CSF outflow resistance rather than an increased rate of CSF production or edema growth.

In all mammalian species, CSF is produced mainly by the choroid plexus located in the ventricular system. After circulating throughout the natural fluid passages of the ventricular system and subarachnoid space, CSF is returned to the vascular system via diverse outflow pathways, including arachnoid granulations (villi) and along the cervical lymphatic system via the olfactory perineural space (see Supplemental Figure 6) [33]. In addition, recent work suggests that part of the CSF is recirculated into the brain via low resistance glymphatic pathways on the surface of the brain that facilitate CSF circulation [34]. It appears likely that the increase in CSF outflow resistance in the pMCAO group found in this study, was the result of impairment of one or more of these CSF absorption pathways.

A progressive increase in ICP can potentially lead to increased high-velocity microvascular shunt (MVS) flow which, in turn, promotes the development of brain edema [35]. In Figure 5a, there is evidence that the slope of the ICP versus infusion rate data changes above $11.7 \mu \mathrm{L} \min ^{-1}$. To avoid this complication, CSF resistance (i.e. the slope) was measured using only the lower infusion rate (low ICP) data, where the brain system has been least perturbed (Fig. 5b).

In summary, these are important results as they demonstrate CSF outflow resistance, not edema, is the mechanism responsible for ICP elevation approximately $24 \mathrm{hrs}$ post-ischemic stroke. This suggests the possibility that intracranial hypertension may be occurring undetected in a much wider range of stroke patients than is currently considered, not just in patients with large malignant strokes and significant levels of brain edema. The results presented here, in conjunction with the work of Professor Sprat and colleagues $[4,12,21,22,36]$ and Professor Baron [1], are consistent with the following mechanism behind unexplained END (see Supplemental Figure 7); First, following ischemic stroke, changes in CSF dynamics, namely increased CSF outflow resistance, can result in a transient elevation of ICP at approximately $24 \mathrm{hrs}$ from stroke onset. Second, these increases in ICP then lead to a progressive reduction in collateral flow and cerebral perfusion in the occluded arterial territory. Third, the tissue that was initially asymptomatic (outside initial ischemic core/penumbra) then becomes at risk and ultimately evolves into infarct, leading to delayed infarct expansion and END. Future work is needed to fully investigate the temporal changes in CSF dynamics and ICP post-ischemic stroke, and how they impact on temporal changes in cerebral blood flow, tissue at risk and infarct evolution. 


\section{Acknowledgments}

We would like to acknowledge the contribution of Dr Robin Young (Robertson Centre for Biostatistics, University of Glasgow) for valuable statistical advice and discussions.

\section{Funding}

MSA would like to acknowledge funding from Ministry of Higher Education in Saudi Arabia.

\section{Compliance with Ethical Standards}

Conflict of Interest: The authors declare that they have no conflict of interest.

Ethical Approval: All applicable international and national guidelines for the care and use of animals were followed. 


\section{References}

1. Seners P, Baron JC. Revisiting 'progressive stroke': incidence, predictors, pathophysiology, and management of unexplained early neurological deterioration following acute ischemic stroke. J Neurol. 2018;265(1):216-25.

2. Seners P, Turc G, Oppenheim C, Baron J-C. Incidence, causes and predictors of neurological deterioration occurring within $24 \mathrm{~h}$ following acute ischaemic stroke: a systematic review with pathophysiological implications. J Neurol Neurosurg Psychiatry [Internet]. 2015;86(1):87-94. Available from: http://jnnp.bmj.com/lookup/doi/10.1136/jnnp-2014-308327

3. Seners P, Turc G, Oppenheim C, Baron J. Incidence, causes and predictors of neurological deterioration occurring within $24 \mathrm{~h}$ following acute ischaemic stroke : a systematic review with pathophysiological implications. 2015;87-94.

4. Beard DJ, Logan CL, McLeod DD, Hood RJ, Pepperall D, Murtha LA, et al. Ischemic penumbra as a trigger for intracranial pressure rise-A potential cause for collateral failure and infarct progression? J Cereb Blood Flow Metab. 2015;36(5):917-27.

5. Kjeldsen SE, Berge E. Blood pressure and early neurological deterioration in acute ischemic stroke. J Hypertens. 2015;33(10):2020-1.

6. Castillo J, Dávalos A, Noya M. Progression of ischaemic stroke and excitotoxic aminoacids. Lancet. 1997;349(9045):79-83.

7. Vila N, Castillo J, Dávalos A, Esteve A, Planas AM, Chamorro Á. Levels of anti-inflammatory cytokines and neurological worsening in acute ischemic stroke. Stroke. 2003;34(3):671-5.

8. Audebert HJ, Pellkofer TS, Wimmer ML, Haberl RL. Progression in lacunar stroke is related to elevated acute phase parameters. Eur Neurol. 2004;51(3):125-31.

9. Frank JI, Frank JI. pressure Large hemispheric infarction, deterioration, and intracranial pressure. 
2011;(July).

10. Read SJ, Hirano T, Abbott DF, Markus R, Sachinidis JI, Tochon-Danguy HJ, et al. The fate of hypoxic tissue on 18F-fluoromisonidazole positron emission tomography after ischemic stroke. Ann Neurol. $2000 ; 48: 228-35$.

11. Gaberel T, Gakuba C, Goulay R, De Lizarrondo SM, Hanouz JL, Emery E, et al. Intracranial Pressure and Collateral Blood Flow. Stroke [Internet]. 2012;9(1):1695-700. Available from: http://www.fluidsbarrierscns.com/content/9/1/3

12. Murtha LA, McLeod DD, Pepperall D, McCann SK, Beard DJ, Tomkins AJ, et al. Intracranial pressure elevation after ischemic stroke in rats: Cerebral edema is not the only cause, and short-duration mild hypothermia is a highly effective preventive therapy. J Cereb Blood Flow Metab [Internet]. 2015;35(4):592600. Available from: http://dx.doi.org/10.1038/jcbfm.2014.230

13. Mann JD, Butler AB, Rosenthal JE, Maffeo CJ, Johnson RN, Bass NH. Regulation of intracranial pressure in rat, dog, and man. Ann Neurol. 1978;3(2):156-65.

14. Marmarou A, Shulman K, Rosende RM. A nonlinear analysis of the cerebrospinal fluid system and intracranial pressure dynamics. J Neurosurg [Internet]. 1978;48(3):332-44. Available from: http://www.ncbi.nlm.nih.gov/pubmed/632857

15. Longa EZ, Weinstein PR, Carlson S, Cummins R. Reversible middle cerebral artery occlusion without craniectomy in rats. Stroke. 1989;20(1):84-91.

16. Hussey F, Schanzer B, Katzman R. A simple constant infusion manometric test for measurement of CSF absorption: II. Clinical studies. Neurology. 1970;20(June):665.

17. Meng X, Fisher M, Shen Q, Sotak CH, Duong TQ. NIH Public Access. 2010;55(2):207-12.

18. Gerriets T, Stolz E, Walberer M, Müller C, Kluge A, Bachmann A, et al. Noninvasive Quantification of Brain Edema and the Space-Occupying Effect in Rat Stroke Models Using Magnetic Resonance Imaging. Stroke. 2004;35(2):566-71.

19. Erdfelder E. $G *$ Power 3 : A flexible statistical power analysis program for the social, behavioral, and 
biomedical sciences. 2007;39(2):175-91.

20. Kotwica Z, H HG, Persson L. Intracranial pressure changes following middle cerebral artery occlusion in rats. 1991;99-104.

21. Beard DJ, Mcleod DD, Logan CL, Murtha LA, Imtiaz MS, Van Helden DF, et al. Intracranial pressure elevation reduces flow through collateral vessels and the penetrating arterioles they supply. A possible explanation for "collateral failure" and infarct expansion after ischemic stroke. J Cereb Blood Flow Metab [Internet]. 2015;35(5):861-72. Available from: http://dx.doi.org/10.1038/jcbfm.2015.2

22. Beard DJ, Murtha LA, McLeod DD, Spratt NJ. Intracranial Pressure and Collateral Blood Flow. Stroke. 2016;47(6):1695-700.

23. Georgiadis D, Schwarz S, Baumgartner RW, Veltkamp R, Schwab S. Influence of Positive End-Expiratory Pressure on Intracranial Pressure and Cerebral Perfusion Pressure in Patients With Acute Stroke. $2015 ; 2088-93$.

24. Lewis PM, Smielewski P, Rosenfeld J V., Pickard JD, Czosnyka M. A Continuous Correlation Between Intracranial Pressure and Cerebral Blood Flow Velocity Reflects Cerebral Autoregulation Impairment During Intracranial Pressure Plateau Waves. Neurocrit Care. 2014;21(3):514-25.

25. Thanvi B, Treadwell S, Robinson T. Early neurological deterioration in acute ischaemic stroke: Predictors, mechanisms and management. Postgrad Med J. 2008;84(994):412-7.

26. Alawneh JA, Jones PS, Mikkelsen IK, Cho T, Siemonsen S, Mouridsen K, et al. tissue after stroke : multivariate modelling of clinical impact. 2011;

27. Roehn-berlage M, Norris DG, Kohno K, Mies G, Leibfritz D, Rossmann K. Evolution of Regional Changes in Apparent Diffusion Coefficient During Focal Ischemia of Rat Brain : The Relationship of Quantitative Diffusion NMR Imaging to Reduction in Cerebral Blood Flow and Metabolic Disturbances. 1995;1002-11.

28. McGarry BL, Jokivarsi KT, Knight MJ, Grohn OHJ, Kauppinen RA. A Magnetic Resonance Imaging Protocol for Stroke Onset Time Estimation in Permanent Cerebral Ischemia. J Vis Exp [Internet]. 2017;(127):1-6. Available from: https://www.jove.com/video/55277/a-magnetic-resonance-imaging- 
protocol-for-stroke-onset-time

29. Kida S, Pantazis A, Weller R. CSF drains directly from the subarachnoid space into nasal lymphatics in the rat . Anatomy, histology and immunological significance. 1993;480-8.

30. Nagra G, Wagshul ME, Rashid S, Li J, McAllister JP, Johnston M. Elevated CSF outflow resistance associated with impaired lymphatic CSF absorption in a rat model of kaolin-induced communicating hydrocephalus. Cerebrospinal Fluid Res. 2010;7:1-8.

31. The I. Changes in Cerebrospinal Fluid Pressure and Outflow from the Lateral Ventricles during Development of Congenital Hydrocephalus in the H-TX Rat. 1987;583:573-83.

32. Orešković D, Klarica M. Measurement of cerebrospinal fluid formation and absorption by ventriculocisternal perfusion: what is really measured? Croat Med J [Internet]. 2014;55(4):317-27. Available from: http://www.ncbi.nlm.nih.gov/pmc/articles/PMC4157383/

33. Sweetman B, Linninger AA. Cerebrospinal fluid flow dynamics in the central nervous system. Ann Biomed Eng. 2011;39(1):484-96.

34. Jessen NA, Finmann Munk AS, Lundgaard I, Nedergaard M. The Glymphatic System - A Beginner's Guide Nadia. Neurochem Res. 2015;40(12):2583-99.

35. Bragin DE, Bush RC, Nemoto EM. Effect of cerebral perfusion pressure on cerebral cortical microvascular shunting at high intracranial pressure in rats. Stroke. 2013;44(1):177-81.

36. Murtha LA, Mcleod DD, Mccann SK, Pepperall D, Chung S, Levi CR, et al. Short-duration hypothermia after ischemic stroke prevents delayed intracranial pressure rise. Int J Stroke. 2014;9(5):553-9. 